\title{
The Current Opinions of Capillary Leak Syndrome
}

\author{
Jun Su${ }^{1}$, Ying Zhang ${ }^{2}$, Wei Hu${ }^{1}$ \\ ${ }^{1}$ Hangzhou First People's Hospital, ICU, Hangzhou, China \\ ${ }^{2}$ The Seventh People's Hospital in Hangzhou, Twelve Wards, Hangzhou, China \\ Email: junjunsu@163.com
}

Received 25 February 2015; accepted 6 March 2015; published 13 March 2015

Copyright (C) 2015 by authors and Scientific Research Publishing Inc.

This work is licensed under the Creative Commons Attribution International License (CC BY). http://creativecommons.org/licenses/by/4.0/

c) (i) Open Access

\begin{abstract}
Capillary Leak Syndrome (CLS) in patients with severe course of disease is more and more common, and the clinical manifestations of CLS include systemic edema, hypoproteinemia, effective circulating blood volume reduction and hemoconcentration. The common pathogenies are sepsis, severe trauma, cardiopulmonary bypass and so on. Clinically, CLS is usually divided into leakage period and recovery period, with different pathophysiologic process, clinical manifestation and treatment in different period respectively. Although there are more treatments, they are not effective treatment measures. There have been so many studies about improvement of endothelial function, macromolecular colloid liquid applications, and continuous blood purification treatment. Systematic understanding of the pathological mechanism, clinical manifestations and staging, diagnosis and treatment of the CLS has a guiding value.
\end{abstract}

\section{Keywords}

Capillary Leak Syndrome, Pathogenesis, Treatment, Continuous Blood Purification

\section{Introduction}

Capillary Leak Syndrome (CLS) is a syndrome of hypoproteinemia, hypovolemic shock, acute renal ischemia in clinical manifestations and the main reasons are the cell injury of capillary endothelial , increased vascular permeability, and a large number of plasma proteins leaking into the tissues of small molecule clearance. [1]. Patients in CLS will be with progressive systemic edema, hypoproteinemia, weight gain, blood concentration rapidly and be multiple organ failure in serious condition.

Diagnostic criteria of CLS [2]: 1) Central venous pressure (CVP) decreases ( $\left.<5 \mathrm{~cm} \mathrm{H}_{2} \mathrm{O}\right)$; 2) No obvious decline of red blood cell specific volume(HCT); 3) Significant reduction of serum albumin $(<25$ g/L); 4) Progressive generalized edema, weight gain or with the chest, pericardial and peritoneal effusion; 5) Oxygenation index 
decreased and X-ray showed exudative changes in interstitial pulmonary. Exclusion criteria: Critically ill patients are after fluid recover and confirmed by cardiac, hepatic, nephrogenic edema, neurogenic pulmonary edema, hemorrhagic and septic shock [3].

Critically ill patients with CLS will influence their prognosis. So incidence, risk factors and the impact on function of the important organs of CLS will get more and more concern by critical medicine physicians. According to reports [4], the main risk factors of CLS are serious infections and surgery in ICU together with SIRS. But critically ill patients' condition in ICU is complex. There are many CLS risk factors and prognosis influence factors. It will be good for physicians to guard against these factors, decrease CLS incidence and mortality and improve CLS patients' prognosis by full understanding of CLS.

\section{Cause and Pathogenesis}

According to internal and external osmotic pressure vessel changes, water and electrolytes can access to the tissue space freely through the capillary barrier and the plasma protein and other ingredients cannot be under normal physiological conditions. Body can cause series of CLS clinical manifestations in the pathological state, for example, severe trauma, sepsis, the operation after cardiopulmonary bypass (particularly in infants and young children) and reperfusion injury, snakebite, acute lung injury or acute respiratory distress syndrome, burns, toxic effects of drugs (recombinant IL-2 and docetaxel) and so on will cause increased capillary permeability by exudating materials of molecular weight less than $20 \times 10^{3} \mathrm{Da}$ and even molecular weight of $90 \times 10^{3} \mathrm{Da}$ in serious condition [5].

\subsection{Serious Infection or Trauma}

These pathogenic factors can cause systemic inflammatory response syndrome by excessive activation of mononuclear macrophage system and release of inflammatory mediator. Under the function of inflammatory mediator, endothelial cell and capillary walls damage, cell junction separation and cracks appear, capillary transport channel diameter and vascular permeability increase, small protein in the blood leaks into the interstitial space by causing the interstitial colloid osmotic pressure, interstitial edema, such as body height of edema, ascites and pleural fluids and the effective circulating blood volume decrease. Interstitial pulmonary edema leads to hypoxemia, tissue hypoxia increases cell damage and then causing MODS [6].

\subsection{The Operation after Cardiopulmonary Bypass}

In the operation of cardiopulmonary bypass, systemic inflammatory response can be caused by the blood surface contact with the pump pipe, non-physiological perfusion, surgical trauma, ischemia reperfusion injury, and changes in body temperature [7]. The inflammation caused by cardiopulmonary bypass is related to perioperative capillary leak. Liu et al. [8] found independent factors of CLS were infection cycle time, type of cardiac malformation, the lowest temperature of operation, age and so on by analysis of 310 cases of congenital heart disease by the operation after cardiopulmonary bypass clinical data of patients.

\subsection{Mechanical Ventilation}

Positive pressure mechanical ventilation itself can cause or worsen lung injury. Slutsky [9] indicated that the mechanical ventilation induced lung injury can demonstrate diffuse alveolar injury (alveolar epithelial and endothelial damage) induced by the gas leakage (i.e. stress injury) and non-cardiac source pulmonary edema.

\subsection{Drug Toxicity}

Using of certain anticancer drugs, especially recombinant human interleukin (interleukin 2, IL-2) therapy, overdose will occur CLS [10] and after injection of interferon $1 \beta$ in patients with multiple sclerosis also can cause CLS. With the increasing use of granulocyte colony-stimulating factor treatment in hematologic cancer and use of arsenic trioxide treatment for recurrence of acute promyelocytic leukemia, the side effects about CLS were increased [11].

\subsection{Inflammatory Mediators}

Oberholzer et al. [12] showed that TNF, IL-1, IL-8 and so on can accelerate the occurrence of CLS. Assier's research shows that rat NK cells involve in the pathological process of CLS [13]. Chihara et al. [5] found that CLS 
patients had increased leukotriene B4 and this might be related to increased vascular permeability, plasma extravasation and others. Rafi found that IL-2 could enhance the cytotoxic T lymphocyte activity, increase fas ligand and perforin, Lymphocyte-activated killer cells and endothelial cells in close contact and start the autolysis to endothelial cell damage. Railan et al. [14] proposed that nitric oxide could lead to vascular endothelial cell injury and increase capillary permeability.

\section{Clinical Manifestations}

CLS can be divided into capillary leakage stage and recovery stage.

\subsection{Capillary Leakage Stage}

\subsubsection{Respiratory System}

With the increased pulmonary capillary permeability, plasma proteins and other small molecules ooze from the vascular permeability to pulmonary interstitial rapidly, pulmonary effusion changes and leads to hypoxemia. Patients in serious condition will be in pulmonary edema [15] and pleural effusion.

\subsubsection{Circulatory System}

Small proteins of plasma leaks to extravascular, plasma colloid osmotic pressure decreases, effective circulating blood volume reduces and then decreased blood pressure may occur. Pericardial effusion as well as cardiac tamponade [16] will format by pericardial effusion increasing and furthermore venous will reduce.

\subsubsection{Digestive System}

Large amount of liquid leaks from the capillary wall and will cause ascites under abdominal wall, gastrointestinal wall and peritoneal edema, then anorexia, nausea, vomiting, abdominal distension, and luminal expansion. Pancreatic tissue edema and trypsin penetrates into the abdominal cavity will make damage increased. Liver cell congestion, edema, degeneration and other pathological changes will impaire liver function and acute liver injury appears.

\subsubsection{Urinary System}

The formation of massive proteinuria will make the plasma colloid osmotic pressure decreased and tissue edema [17] increased further by increased glomerular filtration membrane permeability and plasma protein leakage of small molecules. Low blood volume makes the kidneys perfusion less efficient and also increases the acute kidney injury.

\subsubsection{Nervous System}

Brain capillary permeability increases, a large number of white matter intercellular fluid accumulates (extracellular space expansion) and is rich in protein, glial elements around gray matter blood vessels and neurons swells (glial cell edema), brain size and weight increase, gyrus is wide and flat, sulci becomes narrow, ventricles reduce and the performance of intracranial hypertension appears. Severe cerebral edema can cause herniation [18].

\subsubsection{Mucocutaneous Disorders}

Increased capillary permeability makes small proteins in plasma penetrate into tissue space. And then decreaseing of plasma colloid osmotic pressure, increased interstitial fluid colloid osmotic pressure, serious edema of systemic mucocutaneous, conjunctival edema, increasing tears overflow in plasma samples and pain of limbs muscle show.

\subsection{Convalescence}

In the recovery stage of CLS, there are two ways for interstitial fluid to reflux. When it reabsorbs from the venous capillaries and drains into the right atrium through lymphatic, gradually capillary permeability returns normally and blood volume tends to restore. Leakage of plasma proteins to the organization cannot return to capillaries and the colloid osmotic pressure in tissue fluid is still high because of normal capillary permeability. Edema in patients will hardly disappear with normal serum albumin levels and sometimes may last longer. If continuing to offer a lot of fluid at this time, acute pulmonary edema, alveolar atrophy, gas diffusion barriers, increased arterial and venous blood shunt and decreased arterial partial pressure of oxygen will become. Rapid in- 
creasing in cardiac preload will induce acute left ventricular failure easily and lead to repeated illness. Some studies have shown [19] that the happened pulmonary edema in recovery is more than that in acute leakage. Therefore, giving rehydration therapy should be in the premise of hemodynamic monitoring if condition is permitted.

\section{Treatment}

Currently, there are many researches about CLS therapy with no specific treatment. The following treatment is in favor of shortening the duration of capillary leakage and improving the successful rescue rate except for active treatment and removal of the primary disease inducing predisposing factors.

\subsection{Drug Treatment}

Tahirkhel et al. reported that terbutaline and aminophylline were effective drugs to prevent occurrence of CLS. Terbutaline ( $\beta$ agonist) and aminophylline (phosphodiesterase inhibitor) can loose endothelial cells and improve capillary permeability. In addition, terbutaline has anti-inflammatory effect and can adjust the production of cytokines. Zhang et al. [20] discovered that patients lacked of C4A genes added C4A gene-rich plasma before surgery and it was able to reduce the incidence of CLS after CPB.

\subsection{Fluid Therapy}

The key point of fluid therapy in CLS is maintaining the effective circulating blood volume and avoiding shock. For a long time, liquid crystal or colloid treatment is controversial to CLS patients [21]. With patients' large capillary permeability and small molecular weight of liquid crystals, most of them can leak into the interstitial space and then systemic edema increases after infusion. So we should limit the volume of liquid crystals and avoid interstitial edema and cell swelling caused by excessive liquid crystals in the premise of ensuring the effective circulating blood volume. We need to prevent increased pulmonary edema, cardiac, thoracic and abdominal cavity effusion and added organ damage. Colloid hydroxyethyl starch as the generation of plasma in the traditional product is still the effective and available drug in clinical for CLS treatment. Tian et al. [22] thought that the newest plasma substitutes were hydroxyethyl starch of $130 \times 10^{3}$ Da and $200 \times 10^{3}$ Da molecular weight, polygeline of $(27.5-39.5) \times 10^{3}$ Da molecular weight and so on. In theory, they may have better expansion effect. When blood volume is restored, we use diuretics to reduce swelling of tissues and organs. In the leakage of the CLS, the human serum albumin can leak from capillaries, redistribute after entering into body and influence plasma colloid osmotic pressure in short time. Now we promote to use generation of plasma including new macromolecular plasma substitutes and don't advocate adding FFP and/or human serum albumin for emergency treatment of CLS. At the recovery time, fluid intake should be strictly limited and increased diuresis with normal capillary permeability gradually returning and intravascular volume increasing.

\subsection{Improving Capillary Permeability}

\subsubsection{Glucocorticoid}

It can widely inhibit inflammatory reaction and decrease capillary permeability. In the acute phase, using small doses of hormones can control the inflammatory mediated vascular endothelial injury effectively and reduce the clinical symptoms. It should not be used as a preventive treatment because of the side effects of steroid restrictions.

\subsubsection{Increasing the Amount of EPC}

It can promote injury repair and regeneration of capillaries [23]. Animal experiments showed [24] that increasing the gene expression of vascular endothelial growth factor can specificly increase the concentration of EPC in peripheral blood and promote EPC mobilization and repair of vascular endothelial. Erythropoietin can promote EPC proliferation and differentiation [25]. EPO treatment can increase the number of EPC in peripheral blood and is positively correlated with concentration of erythropoietin.

\subsubsection{Histamine Dihydrochloride}

It can protect IL-2 induced rat model of CLS by dose-dependent and reduce the CLS severity and mortality [26]. Comparing with $42 \%$ survival rate of the control group, the dose of histamine dihydrochloride are $0.47,4.7,47.0$ $\mathrm{mg} / \mathrm{kg}$ and the model mouse survival rates are $56 \%, 75 \%$ and $81 \%$. It can reduce the IL-2-induced lung injury, decrease pulmonary edema (lung wet and dry ratio reduce up to 62\%) and pulmonary capillary leakage deter- 
minated by Evans blue dye.

\subsubsection{Interferon- $\beta$}

Studies have shown that [27] pretreatment of Interferon- $\beta$ (IFN- $\beta$ ), CD73's new type inducer could significantly improve the barrier function of animals after intestinal ischemia-reperfusion pulmonary vascular. Interferon- $\beta$ can almost completely inhibit capillary leakage after ischemia in reperfusion period obviously. Interferon- $\beta$ can induce CD73 expression of pulmonary vascular endothelial cells in cultured human and reduce capillary leakage meanwhile. So it is expected to be used in clinical treatment of CLS.

\subsection{Respiratory Support}

In the leakage of CLS, for a large number of fluid in capillaries leaks to interstitial lung with making lung compliance, increased airway resistance, decreased ventilatory function and severe hypoxemia may occur. Mechanical ventilation improves lung compliance and ventilation or perfusion ratio, decreases airway resistance, improves oxygenation and reduces pulmonary effusion by increasing positive end expiratory pressure. Mechanical ventilation can effectively increase the oxygen supply of tissues and organs and has great help in improving body environment and treatment of primary disease [28].

\subsection{Continuous Blood Purification}

CBP treatment can enhance clearance of inflammatory mediators, improve pulmonary gas exchange, keep hemodynamics stable control of azotemia, reduce fluid overloading because of entering macromolecular synthetic colloids, be convenient for nutrition support, improve oxygen metabolism or tissue perfusion in patients [29], control SIRS symptoms, prevent the development of the disease to the MOD and improve the survival rate in critically ill patients. It also can make rapid absorption and removal of endotoxin in blood, correct acidosis and improve the body's homeostasis. Meanwhile, CBP has a protective effect on vascular endothelial function [30]. CBP also can improve coagulation dysfunction in vivo because of heparin. It can reduce blood coagulation cascade [31] and play a role in the treatment CLS.

\section{Conclusion}

Currently, there is no specific prevention and treatment for critically ill patients in CLS. We should restore blood volume as soon as possible, improve hypoxemia and capillary permeability and reduce the degree of capillary leakage at the same time of active treatment of primary disease. With the pathogenesis deepening research of CLS and the comprehensive application of various prevention methods, the incidence of CLS will reduce, the survival rate will increase and patients' prognosis will improve.

\section{References}

[1] Lee, Y.S., Kim, S.Y., Kwon, C.W., Song, H.G., Lee, Y.K., Kim, H.J., et al. (2007) Two Cases of Systemic Capillary Leak Syndrome That Were Treated with Pentastarch. The Korean Journal of Internal Medicine, 22, 130-132. http://dx.doi.org/10.3904/kjim.2007.22.2.130

[2] Marx, G., Vangerow, B., Burczyk, C., Gratz, K.F., Maassen, N., Cobas Meyer, M., et al. (2000) Evaluation of Noninvasive Determinants for Capillary Leakage Syndrome in Septic Shock Patients. Intensive Care Medicine, 26, 12521258. http://dx.doi.org/10.1007/s001340000601

[3] Stiller, B., Sonntag, J., Dahnert, I., Alexi-Meskishvili, V., Hetzer, R., Fischer, T., et al. (2001) Capillary Leak Syndrome in Children Who Undergo Cardiopulmonary Bypass Clinical Outcome in Comparison with Complement Activation and C1 Inhibitor. Intensive Care Medicine, 27, 193-200. http://dx.doi.org/10.1007/s001340000704

[4] Zhang, Y.L. and Wan, X.R. (2006) Capillary Leak Syndrome. Chinese Journal of Postgraduates of Medicine, 29, 10-13.

[5] Chihara, R., Nakamoto, H., Arima, H., Moriwaki, K., Kanno, Y., Sugahara, S., et al. (2002) Systemic Capillary Leak Syndrome. Internal Medicine, 41, 953-956. http://dx.doi.org/10.2169/internalmedicine.41.953

[6] Feng, X., Ren, B., Xie, W., Huang, Z., Liu, J., Guan, R., et al. (2006) Influence of Hydroxyethyl Starch 130/0.4 in Pulmonary Neutrophil Recruitment and Acute Lung Injury during Polymicrobial Sepsis in Rats. Acta Anaesthesiologica Scandinavica, 50, 1081-1088. http://dx.doi.org/10.1111/j.1399-6576.2006.01113.x

[7] St. Yang, G.N., Tang, B.Y., Xiong, W.P., et al. (2007) Hydroxyethyl Starch 130/0.4 in Patients after Cardiopulmonary Bypass Cytokines. Journal of Clinical Healthcare, 10, 367-369. 
[8] Liu, C.J., Liu, L., Xu, F., et al. (2006) Children with Congenital Heart Disease, Capillary Leak Syndrome after Cardiopulmonary Bypass Influencing Factors. Chinese Journal of Practical Pediatrics, 21, 753-755.

[9] Slutsky, A.S. (1999) Lung Injury Caused by Mechanical Ventilation. Chest, 116, 9S-15S. http://dx.doi.org/10.1378/chest.116.suppl_1.9S-a

[10] Zhang, L. and Ning, X.H. (2004) Dose IL-2 Caused 1 Case of Adverse Reactions. Chinese Academy of Medical Sciences, 26, 236.

[11] Guo, L.Y., Zhao, X.T. and Li, W. (2005) Arsenic Trioxide Treatment of Relapsed Acute Promyelocytic Leukemia Complicated with Capillary Leak Syndrome. Journal of Internal Medicine, 44, 632.

[12] Oberholzer, A., Oberholzer, C., Moldawer, L.L., Luce, J.M., Yaffe, M.B. and Fink, M.P. (2000) Cytokine SignalingRegulation of the Immune Response in Normal and Critically Ill States. Critical Care Medicine, 28, N3-N12. http://dx.doi.org/10.1097/00003246-200004001-00002

[13] Assier, E., Jullien, V. and Lefort, J. (2005) Constitutive Expression of IL-2Rbeta Chain and Its Effects on IL-2-Induced Vascular Leak Syndrome. Cytokine, 32, 280-286. http://dx.doi.org/10.1016/j.cyto.2005.10.006

[14] Railan, D., Fivenson, D.P. and Wittenberg, G. (2000) Capillary Leak Syndrome in a Patient Treated with Interleukin 2 Fusion Toxin for Cutaneous T-Cell Lymphoma. Journal of the American Academy of Dermatology, 43, 323-324. http://dx.doi.org/10.1067/mjd.2000.106373

[15] Ma, L.J. and Qin, Y.Z. (2008) Extravascular Lung Water Index and Pulmonary Capillary Permeability Index in the Diagnosis of Pulmonary Edema. Chinese Science Abstracts, 14, 195-196.

[16] Wang, B. and Guo, J.H. (2004) Cardiac Tamponade. Journal of Cardiac Arrhythmias, 8, 38-39.

[17] Long, J. (2010) Nephrotic Syndrome 80 Cases. Journal of Clinical Rational Drug Use, 3, 96-97.

[18] Li, Q., Lu, B.-X. and Wang, Z.J. (2007) Selegiline Treatment of Vasogenic Brain Edema in Experimental Study. Practical Journal of Medicine, 24, 1344-1346.

[19] Schwartzentruber, D.J. (2001) Guidelines for the Safe Administration of High-Dose Interleukin-2. Journal of Immunotherapy, 24, 287-293. http://dx.doi.org/10.1097/00002371-200107000-00004

[20] Zhang, S., Wang, S., Li, Q., Yao, S.L., Zeng, B.H., Ziegelstein, R.C. and Hu, Q.H. (2005) Capillary Leak Syndrome in Children with C4A-Deficiency Undergoing Cardiac Surgery with Cardiopulmonary Bypass: A Double-Blind, Randomized Controlled Study. The Lancet, 366, 556-562. http://dx.doi.org/10.1016/S0140-6736(05)67099-7

[21] Marx, G. (2003) Fluid Therapy in Sepsis with Capillary Leakage. European Journal of Anaesthesiology, 20, 429-442. http://dx.doi.org/10.1097/00003643-200306000-00002

[22] Tian, Z.M., Jin, T., He, H.Y., et al. (2005) Critically Ill Patients with Capillary Leak Syndrome. Critical Care Medicine, 25, 333-335.

[23] Tichelli, A. and Gratwohl, A. (2008) Vascular Endothelium as "Novel” Target of Graft-Versus-Host Disease. Best Practice Research Clinical Haematology, 21, 139-148. http://dx.doi.org/10.1016/j.beha.2008.02.002

[24] Kalka, C., Masuda, H., Takahashi, T., Gordon, R., Tepper, O., Gravereaux, E., et al. (2000) Vascular Endothelial Growth Factor $_{165}$ Gene Transfer Augments Circulating Endothelial Progenitor Cells in Human Subjects. Circulation Research, 86, 1198-1202. http://dx.doi.org/10.1161/01.RES.86.12.1198

[25] Bahlmann, F.H., De Groot, K., Duckert, T., Niemczyk, E., Bahlmann, E., Boehm, S.M. et al. (2003) Endothelial Progenitor Cell Proliferation and Differentiation Is Regulated by Erythropoietin. Kidney International, 64, 1648-1652. http://dx.doi.org/10.1046/j.1523-1755.2003.00279.x

[26] Gilman, A.L., Ozkaynak, M.F., Matthay, K.K., Krailo, M., Yu, A.L., Gan, J., et al. (2009) Phase I Study of ch14.18 with Granulocyte-Macrophage Colony-Stimulating Factor and Interleukin-2 in Children with Neuroblastoma after Autologous Bone Marrow Transplantation or Stem-Cell Rescue: A Report from the Children's Oncology Group. Journal of Clinical Oncology, 27, 85-91. http://dx.doi.org/10.1200/JCO.2006.10.3564

[27] Kiss, J., Yegutkin, G.G., Koskinen, K., Savunen, T., Jalkanen, S. and Salmi, M. (2007) IFN- $\beta$ Protects from Vascular Leakage via Up-Regulation of CD73. European Journal of Immunology, 37, 3334-3338. http://dx.doi.org/10.1002/eji.200737793

[28] Zheng, X., Liu, B.-Y., Fang, Z.-C., et al. (2011) Effects of Cell Apoptosis on Ventilator-Induced Lung Injury. Journal of Southeast University (Medical Science Edition), 30, 360-363.

[29] Chrysochoou, G., Marcus, R.J., Sureshkumar, K.K., McGill, R.L. and Carlin, B.W. (2008) Renal Replacement Therapy in the Critical Care Unit. Critical Care Nursing Quarterly, 31, 282-290. http://dx.doi.org/10.1097/01.CNQ.0000336813.04548.22

[30] Wang, J.Q., Tao, X.G., Zhang, C.-P., et al. (2007) Continuous Blood Purification in Severe Sepsis Patients with Endothelial Injury. Journal of Emergency Medicine, 16, 514-517.

[31] Lao, Z.-G., Xiong, X.-L., Wu, H., et al. (2011) Observation on Different Blood Purification Modes for Multiple Organ Dysfunction Syndrome Patients Associated with Acute Renal Failure. Modern Medical Journal, 39, 178-180. 